\title{
Micro and Nano-CT as a Valuable and Complimentary Tool for Life Science Research
}

\author{
M.E. Faillace ${ }^{1}$, R. A. Rudolph ${ }^{1}$, O. Brunke ${ }^{2}$ \\ ${ }^{1}$ GE Inspection Technologies, LP., 50 Industrial Park Road, Lewistown, PA 17044 USA \\ ${ }^{2}$ GE Sensing \& Inspection Technologies GmbH, Niels-Bohr-Straße 7, 31515 Wunstorf, Germany
}

X-ray computed tomography (CT) has long been an established technique used for diagnostic purposes in the healthcare arena and extensively used for life science research applications; for example in the orthopedic, geological, and paleontology fields. Micro $(\mu \mathrm{CT})$ and nano-CT allow for three dimensional nondestructive examination of sample morphology and density at high spatial and contrast resolution [1]. Recent advancements in $\mathrm{x}$-ray tube technology, such as micro and nano-focus open x-ray tubes, paired with efficient flat panel detectors has led to the development of compact cabinet CT systems that achieve spatial resolution of sub 1 micron $(\mu \mathrm{m})$. Moreover, these developments allow for high photon flux at very small focal spot sizes and provide excellent contrast resolution and signal to noise ratios similar to those achieved in synchrotron radiation-based $\mu \mathrm{CT}$ [2].

Micro and nano-CT are viable inspection tools for biological applications as they can be used to compliment medical imaging techniques at increased resolution in the absence of dose restrictions. Due to its non-destructive nature, CT samples can be further utilized for other experimental techniques such as mechanical testing and histology. In the following studies we describe micro and nano-CT results from several biological samples. The data demonstrate advancements in signal-to-noise, contrast and spatial resolution, sharpness and sample penetration as well as showing the wide-reaching applications of CT technology in the life sciences arena.

GE has worked collectively with many institutions in fields such as orthopedic, biological and anthropological research to scan a wide variety of biological samples in order to demonstrate the value of this tool to life sciences research. A sampling of the data described here include, tissue from fetal and adult mice, a hover fly, a soil/plant interface, and a nummulite. Minimal to no sample preparation was required for scanning.

Mouse samples were harvested during necropsy, following Institutional Animal Care and Use Committee (IACUC) approval. Samples were stored in PBS and frozen. Prior to data collection samples were thawed and scanned in fresh PBS. The rat knee joint was harvested at necropsy and prepared with microfill, an x-ray opaque material, which allowed imaging of the vasculature.

Images were collected on GE phoenix|x-ray nanotom $\mathrm{m}$ and vltomelx $\mathrm{m}$ systems, which have been newly introduced to the commercial market. Images were generated using previously described techniques [2] and extracted 2D and 3D visualizations are presented here.

This study demonstrates that innovations in system technology enable improvements in spatial and contrast resolution, sample penetration and overall image quality. This allows imaging of morphological and density features within biological samples that have previously gone unexplored. 
The degree of mineralization of 1-day fetal mouse bones is approximately $36 \%$ of adult mineralization values [3]. Here, we have shown that imaging of tissue that contains a low level of mineral is possible due to advances in signal-to-noise ratio, contrast and spatial resolution. Additionally, qualitative assessment of the data resolves individual bones, on the order of $0.5 \mathrm{~mm}$ and below.

Enhanced contrast resolution allowed imaging of bone and soft-tissue vasculature in the rat knee at high resolution in a lower energy range. 3-D data reveals the spatial mapping of the vasculature in three dimensions allowing for more precise sectioning of samples for further analysis with minimal damage and material loss [4]. Image quality such as this is possible due to advances in detector technology. Flat panel detectors are built on an amorphous silicon (a-Si) panel with a CsI scintillator deposited as needle structure. Molybdenum targets further enhance imaging at lower energy ranges. These features help to minimize beam hardening artifacts created by incomplete sample penetration by lower energy x-rays.

Historically, CT technology has been widely used in the healthcare field and adapted for extended use in life sciences research. Recent improvements to the technology have increased image resolution, allowing analysis of morphology that has previously gone unexplored. Improvements in high performance industrial cabinet sub- $\mu \mathrm{CT}$ technologys allows the use of CT in life sciences to adequately support and complement research projects where high quality imaging data is required. Application results from these new technologies demonstrate a high potential for usage in innovative biological research and analyses, metrology and morphometric studies.

References: [1] Ebel D.S. et al. 2007. Meteoritics \& Planetary Science 42:1627-1646 [2] Kastner J. et al. 2010. NDT\&E Int. 43: 599-605 [3] Miller L.M et al. 2007. JBMR 22: 1037-1045 [4] Ebel D. S. et al. 2011. Abstract. Meteoritical Society Meeting . Meteoritics \& Planetary Science 43:1725-1740
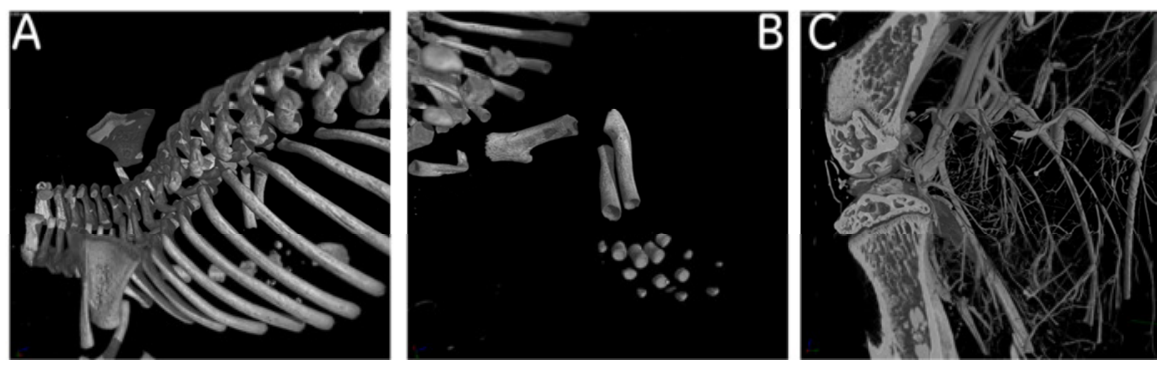

Fig. 1. 3D renderings of 1 day old mouse (A) rib, spine, and (B) paw. (C) Adult rat knee showing bones and vasculature. Samples courtesy of Karl Jepsen, University of Michigan.
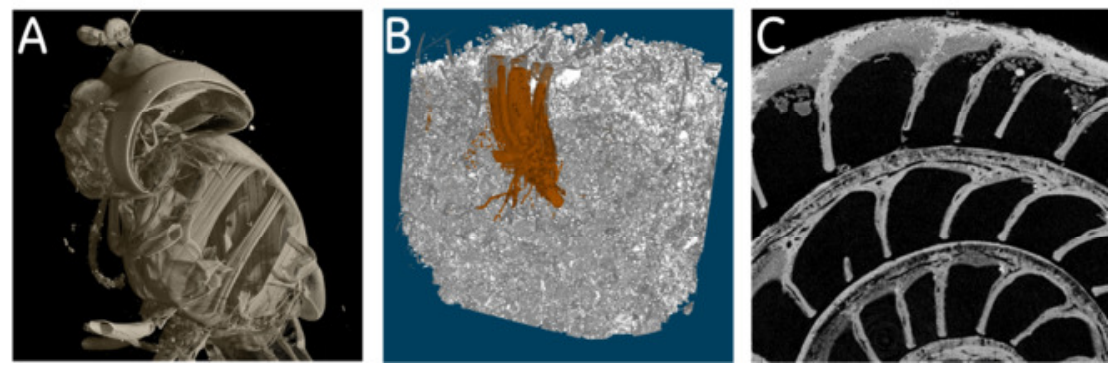

Fig. 2. (A) Cross-section of Hover fly, eye facet structures identifiable. (B) Soil aggregate with segmented grass roots grown in clay-based soil. (C) Slice view of a Nummulite Courtesy of R. Speijer, K.U. Leuven, Belgium 